\title{
Resultados clínicos e de imagem da abordagem da lesão de Hill-Sachs pela técnica de remplissage na instabilidade anterior do ombro*
}

\section{Clinical and Image Outcomes of the Hill-Sachs Injury Approach by the Remplissage Technique on the Anterior Shoulder Instability}

\author{
Flávio de Oliveira França ${ }^{1,2}$ André Couto Godinho ${ }^{1,2,3}$ Diego Pedrosa Capitol Carneiro Leal ${ }^{1,2}$ \\ Mateus Matos Mantovani ${ }^{1,2}$ Rafael Rodrigues Frazão ${ }^{1,2}$ Ricardo Ferreira Mariz ${ }^{1,2}$
}

${ }^{1}$ Hospital Ortopédico, Belo Horizonte, MG, Brasil

2 Hospital Belvedere, Belo Horizonte, MG, Brasil

${ }^{3}$ Hospital Belo Horizonte, Belo Horizonte, MG, Brasil

\author{
Address for correspondence Flávio de Oliveira França, Hospital \\ Ortopédico, Belo Horizonte, MG 30210-300, Brasil \\ (e-mail: flavio.franca29@gmail.com).
}

Rev Bras Ortop 2019;54:13-19.

\begin{abstract}
Resumo
Palavras-chave

- ombro

- instabilidade articular

- recidiva

- lesões de Bankart

- artroscopia

Objetivos Avaliar o resultado funcional da técnica de remplissage, a cicatrização da capsulotenodese do tendão infraespinal no defeito de Hill-Sachs, o grau de infiltração gordurosa do músculo infraespinal e sua força de rotação lateral no pós-operatório. Método Foram avaliados 25 pacientes com luxação anterior recidivante do ombro e lesão de Hill-Sachs com índice de Hardy maior do que $20 \%$, submetidos à técnica artroscópica de remplissage com seguimento mínimo de um ano. Os pacientes foram submetidos a avaliação clínica (escores funcionais de Carter-Rowe e Walch-Duplay, medição de amplitude de movimento e força) e exame de ressonância magnética no ombro operado.

Resultados Dos pacientes, $88 \%$ e $92 \%$ apresentaram resultados bons ou excelentes nas avaliações funcionais pelos escores de Carter-Rowe e Walch-Duplay, respectivamente. Identificou-se diferença média de $1 \mathrm{~kg}$ a menos de força do membro operado em relação ao contralateral $(p<0,001)$ e diferença média de 10 o em rotação lateral $1 \mathrm{e}$ $2(p<0,001)$, novamente com o uso como referência do lado contralateral. Todos os pacientes submetidos a ressonância magnética apresentaram preenchimento de alto grau da lesão de Hill-Sachs pela capsulotenodese, assim como ausência ou mínima infiltração gordurosa no músculo infraespinal.

Conclusão A técnica de remplissage apresentou resultados bons/excelentes nos escores funcionais, apesar da perda discreta de força e amplitude de rotação lateral com significância estatística. Foram observados resultados excelentes quanto à cicatrização da capsulotenodese e ao preenchimento do defeito de Hill-Sachs.
\end{abstract}

Trabalho desenvolvido no Hospital Ortopédico, Belo Horizonte, MG, Brasil. Publicado originalmente por Elsevier Editora Ltda. (c) 2018 Sociedade Brasileira de Ortopedia e Traumatologia.

(1) Flávio de Oliveira França's ORCID is https://orcid.org/0000-00029632-2666.

received

July 25, 2017

accepted

October 24, 2017

published online

February 7, 2018
DOI https://doi.org/

10.1016/j.rbo.2017.10.010. ISSN 0102-3616.
Copyright $\odot 2019$ by Sociedade Brasileira License terms de Ortopedia e Traumatologia. Published by Thieme Revnter Publicações Ltda, Rio de Janeiro, Brazil 


\begin{abstract}
Keywords

- shoulder

- joint instability

- recurrence

- Bankart lesions

- arthroscopy

Objective To evaluate the functional outcome of the remplissage technique, the healing of the capsulotenodesis of the infraspinatus tendon in Hill-Sachs lesion, and the degree of fatty infiltration of the infraspinatus muscle and its postoperative strength.

Methods Twenty-five patients with recurrent anterior dislocation of the shoulder and Hill-Sachs lesion with a Hardy index $>20 \%$ who underwent the remplissage arthroscopic technique were evaluated with a minimum follow-up of 1 year. Patients underwent a clinical evaluation (Carter-Rowe and Walch-Duplay functional scores, measurement of range of motion and strength) and a magnetic resonance imaging (MRI) exam on the operated shoulder.

Results Eighty-eight percent and $92 \%$ of the patients had good or excellent scores in the functional assessments of the Carter-Rowe and Walch-Duplay scores, respectively. A mean difference of $-1 \mathrm{~kg}$ in the strength of the operated limb was observed when compared with the contralateral $\operatorname{limb}(p<0.001)$, as well as a mean difference of $10^{\circ}$ in external rotation 1 and $2(p<0.001)$, also compared with the contralateral side. All of the patients who underwent an MRI exam presented high-grade filling of the Hill-Sachs lesion by capsulotenodesis, as well as absence of or minimal fatty infiltration in the infraspinatus muscle. Conclusion The remplissage technique had good/excellent functional score results, despite the discrete, albeit statistically significant, loss of strength and of external rotation amplitude. Successful capsulotenodesis healing and filling of the Hill-Sachs defect were demonstrated.
\end{abstract}

\section{Introdução}

A lesão posterossuperior da cabeça do úmero associada à luxação glenoumeral foi descrita por Hill e Sachs em $1940^{1} \mathrm{e}$ desde então tem ganhado importância crescente na avaliação da instabilidade anterior do ombro. Sua incidência de $47 \%$ após primoluxações pode aumentar para até $84 \%$ nos quadros de luxação anterior recidivante ${ }^{2,3}$ e sua participação na recidiva das luxações anteriores e na falha do tratamento após reparo isolado da lesão essencial de Bankart já é amplamente reconhecida.

Burkhart e De Beer ${ }^{4}$ descreveram o termo engaging HillSachs para as fraturas por impacção que, por sua profundidade e orientação, permitem seu encaixe na borda da glenoide na posição de abdução e rotação lateral. Yamamoto et al. ${ }^{5}$ aprimoraram esse conceito através do glenoid track, que permitiu avaliar, com o uso de estudo tomográfico préoperatório, se a lesão de Hill-Sachs se expõe ou não ao risco de engaging na borda da glenoide.

Junto à evolução de sua importância e diagnóstico, desenvolveu-se também sua abordagem terapêutica. Nesse contexto, Wolf et al. ${ }^{6}$ descreveram a técnica artroscópica de preenchimento da lesão com cápsula posterior e tendão do infraespinal, tornaram-na extra-articular e impediram o engaging. Essa técnica foi intitulada remplissage, que, em português, significa preenchimento.

O presente estudo tem por objetivo:

- Avaliar o resultado funcional da técnica de remplissage;

- Avaliar a cicatrização da capsulotenodese no defeito de Hill-Sachs;

- Avaliar o grau de infiltração gordurosa do músculo infraespinal no pós-operatório;
- Avaliar força de rotação externa do ombro no pósoperatório.

Nossa hipótese é que essa técnica promove cicatrização satisfatória da capsulotenodese posterior e excelentes resultados clínicos sem grandes repercussões na amplitude de movimento do ombro, força e qualidade do músculo infraespinal.

\section{Material e Métodos}

\section{Seleção de Pacientes}

Estudo retrospectivo analítico que abordou indivíduos submetidos à cirurgia de reparo artroscópico da lesão de Bankart associada à técnica de remplissage para tratamento da instabilidade anterior do ombro.

Entre 2009 e 2015, 34 pacientes (34 ombros) foram submetidos ao reparo artroscópico da instabilidade anterior do ombro pela técnica de remplissage pelos cirurgiões do Grupo de Ombro do Hospital Ortopédico de Belo Horizonte. Desses, nove não foram localizados ou não concordaram em participar do presente estudo. 0 seguimento mínimo foi de 12 meses e o máximo de 84 , com média de 33,3 . A média de idade no ato do procedimento foi de 32,2 anos (de 18-51), o membro dominante foi abordado em $60 \%$ dos casos. Quanto ao gênero, $20 \%$ dos pacientes eram do sexo feminino. A média de idade no primeiro evento de instabilidade foi de $24 \pm 6$ anos, $88 \%$ tinham apresentado pelo menos um episódio de luxação (necessitaram de manobra de redução em atendimento médico) e $24 \%$ dos pacientes apresentaram subluxações recidivantes. Entre os pacientes que souberam informar o número de episódios de luxação, a média foi de dois episódios antes do procedimento. $\mathrm{O}$ intervalo médio entre 
o primeiro evento de instabilidade e o procedimento foi de cinco anos.

Foram incluídos pacientes com diagnóstico de luxação anterior recidivante do ombro, de origem traumática, com lesão de Bankart associada a lesão de Hill-Sachs, evidenciadas em ressonância magnética (RM), com índice de Hardy mínimo de $20 \%$ medido em tomografia computadorizada. Foram excluídos aqueles pacientes com lesões do manguito rotador (MR) associadas, assim como fraturas do terço proximal do úmero ou Bankart ósseo maior do que 20\% e seguimento pós-cirúrgico menor do que um ano.

\section{Técnica Cirúrgica}

Sob anestesia geral e bloqueio do plexo braquial, o paciente é colocado na posição de decúbito contralateral, com o tronco inclinado 30。 para posterior e membro acometido com abdução de aproximadamente 50 e flexão anterior de 15。, sob tração axilar vertical de $7 \mathrm{~kg}$ e longitudinal fixa. ${ }^{7}$

0 acesso à articulação glenoumeral é feito através de portal posterior tradicional, $2 \mathrm{~cm}$ medial e $2 \mathrm{~cm}$ inferior ao ângulo posterolateral do acrômio. Para a visualização da lesão de Hill-Sachs é retirada a tração vertical e aumentada a tração longitudinal com objetivo de abrir o espaço entre o deltoide e o tubérculo maior do úmero. É feito portal percutâneo distal à borda lateral do acrômio, cuja localização é determinada com agulha de punção para posicioná-lo em direção ao ponto central da lesão de Hill-Sachs. Faz-se cruentização sem aprofundamento da lesão, usa-se lâmina óssea de shaver. Em seguida, colocação de uma ou duas âncoras de $5 \mathrm{~mm}$, de acordo com o tamanho do defeito ósseo (-Fig. 1). Preferencialmente, usamos duas âncoras, uma posicionada na margem superomedial e outra na inferomedial da lesão, com aproximadamente $1 \mathrm{~cm}$ de distância mínima entre elas. Deve-se evitar a colocação das âncoras com mais de $2 \mathrm{~cm}$ de distância, sob risco de envolvimento do músculo redondo menor, inferiormente. ${ }^{8}$ Passam-se os fios de sutura através da cápsula posterior e tendão do músculo infraespinal, com auxílio do penetrator, prepara-se o ponto em $U$, são deixados reparados para ser suturados ao fim do

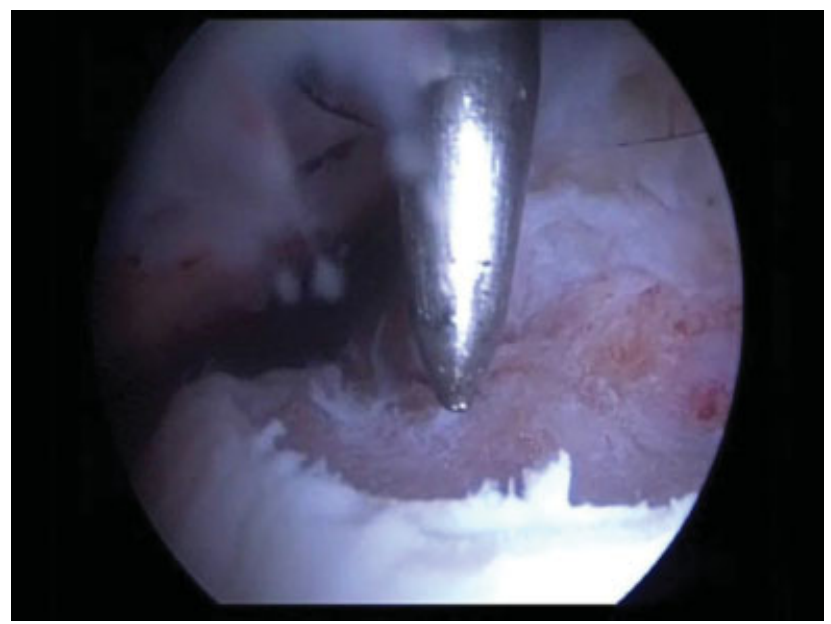

Fig. 1 Posicionamento da âncora na lesão de Hill-Sachs.

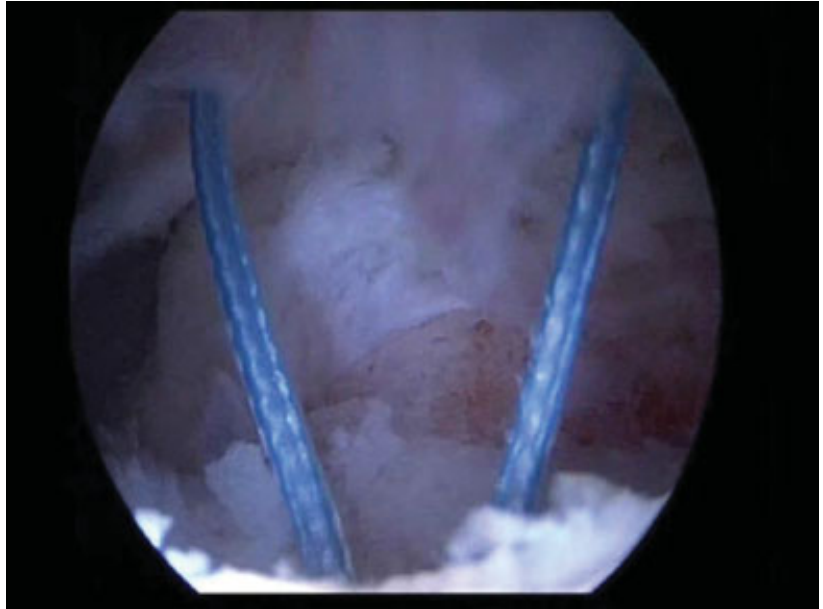

Fig. 2 Preparo do ponto em U para capsulotenodese.

procedimento (-Fig. 2). Os portais anteroinferior e anterossuperior são confeccionados pelo método inside-out, através do intervalo rotador, na borda superior do tendão subescapular e na margem anterior do acrômio adjacente ao tendão da cabeça longa do bíceps, respectivamente, são mantidos por cânulas 8,5 mm. Passa-se, então, o artroscópio, do portal posterior para a cânula anterossuperior, coloca-se uma cânula de 7,5 mm no portal posterior para instrumentação.

Faz-se o reparo padrão da lesão de Bankart, com âncoras nas posições 1 , 3 e 5 hour para ombro direito, ou o equivalente 7, 9 e 11 hour para ombro esquerdo, introduzidas na borda anterior da glenoide, com máximo de $2 \mathrm{~mm}$ de medialização articular. Então, é feita a capsulotenodese na lesão de Hill-Sachs, fixam-se os pontos em U previamente passados. Fazemos a visualização intra-articular do preenchimento da lesão de Hill-Sachs nesse momento (visualização indireta da sutura) (-Fig. 3).

\section{Pós-operatório}

Imobilização com tipoia do tipo coxim de abdução, por seis semanas, para manutenção de rotação neutra, evita-se estresse

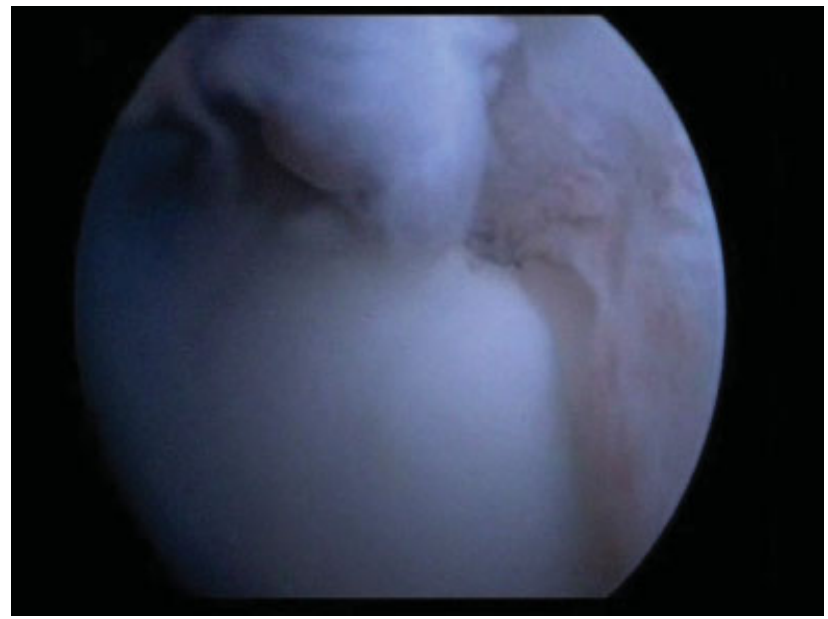

Fig. 3 Visualização intra-articular do preenchimento da lesão de Hill-Sachs. 
na capsulotenodese, são liberadas flexão e extensão ativas do cotovelo, punho e dedos, a partir do primeiro dia de pósoperatório e movimentos pendulares do ombro a partir da segunda semana. Iniciada reabilitação fisioterápica, dividida em três fases: a primeira, com analgesia e ganho de amplitude de movimento (ADM) passivo e autopassivo; na segunda, iniciase fortalecimento muscular isométrico do manguito rotador; por último, é feito fortalecimento muscular isotônico. Treinamento de propriocepção progressivo desde a primeira fase.

\section{Avaliação Clínica e Funcional}

A avaliação clínica deu-se através de perguntas direcionadas a determinar características demográficas e específicas do quadro clínico de instabilidade. A avaliação funcional se deu por meio dos escores de Carter-Rowe ${ }^{9}$ e Walch-Duplay, ${ }^{10}$ que levam em consideração critérios como: estabilidade, com avaliação de apreensão, subluxação ou recidiva de luxação; mobilidade, é avaliada ADM em elevação anterior, rotação lateral e medial; função, questionam-se limitações para trabalho e/ou esportes e dor. Com o protocolo pré-determinado, ${ }^{11}$ foram avaliadas as amplitudes de movimento com auxílio de goniômetro e força de rotação lateral com cotovelo em flexão de 90 graus e braço em neutro, com auxílio de balança de uso doméstico.

\section{Avaliação de Imagem Pós-operatória}

\section{Avaliação Fisor e Goutallier}

Ao fim da avaliação, foi sugerido aos pacientes que fizessem exames de ressonância magnética dos ombros pós-operados para a análise do preenchimento da lesão de Hill-Sachs e da infiltração gordurosa do músculo infraespinal. Foi usada como base a classificação Fisor (escore do índice de preenchimento do remplissage), ${ }^{12}$ que classifica o preenchimento, avaliado nos cortes axial e sagital, em cinco graus: preenchimento completo (4 pontos), parcial com defeito residual pequeno (3 pontos), parcial com defeito residual grande (2 pontos), preenchimento mínimo (1 ponto) e completa falha do preenchimento (0 ponto). Por fim, deve ser feito o somatório de pontos dos cortes axial e sagital, dividem-se em cinco grupos de resultado: excelente (7-8 pontos), bom (5-6 pontos), regular ( $3-4$ pontos), ruim (1-2 pontos) e ausência de preenchimento (zero ponto).

\section{Adaptação ao Fisor}

Como nossa avaliação levou em consideração pacientes submetidos à RM sem contraste (negativa dos pacientes de fazer o exame), posição do braço em rotação lateral e avaliação das imagens somente na sequencia axial, levamos em consideração somente a metodologia de classificação final do Fisor. O objetivo de fazer o exame em rotação lateral foi de relaxar o tendão do infraespinal e tornar mais evidente uma possível falha na cicatrização.

Ainda referente à nossa avaliação da cicatrização da capsulotenodese, que originalmente se classificaria em cinco grupos, fez-se necessário o agrupamento em baixo grau de preenchimento (Fisor 0 a 2) e alto grau de preenchimento (Fisor 3 a 4), a fim de aprimorar a análise estatística.

\section{Análise Estatística}

As variáveis foram testadas em relação ao tipo de distribuição pelo teste de Kolmogorov-Smirnov, as variáveis de força e rotação lateral foram comparadas pelo teste $t$ de Student pareado. $O$ nível de significância considerado foi de $p<0,05$.

O trabalho teve a aprovação do Comitê de Ética sob o número CAAE 62694016.7.0000.5125.

\section{Resultados}

\section{Resultados Funcionais}

A média do escore de Carter-Rowe foi de $91 \pm 14$ (variou de 50 a 100) com $88 \%$ de bons e excelentes resultados ${ }^{9}$ e a média do escore Walch-Duplay foi de $92 \pm 10$ (variou de 70 a 100) com $92 \%$ de bons e excelentes resultados ${ }^{10}$ (- Tabelas 1 e 2 ).

$\mathrm{Na}$ força de rotação lateral, houve uma diferença média de $-0,92 \mathrm{~kg}$ quando comparado com o ombro contralateral (-Tabela 3).

Quanto à amplitude de movimento, houve diferença média de 10 o quando considerada a rotação lateral com 0 。 de abdução (RL1) e de 10。 quando considerada a rotação lateral com 90。 de abdução (RL2), novamente quando comparada com o ombro contralateral ( - Tabela 4).

Com relação à recidiva de instabilidade, obtivemos quatro pacientes (16\%) com exame clínico de apreensão positivo e somente um (4\%) com queixa de episódio de subluxação pósoperatória, nenhum dos pacientes avaliados apresentou episódio de luxação pós-operatória.

\section{Resultados de Imagem}

Dos 25 pacientes avaliados, 16 concordaram em fazer uma ressonância magnética de controle, de acordo com a adaptação do escore Fisor ${ }^{12}$ todos apresentaram preenchimento de alto grau ( - Fig. 4A e B) (-Tabela 5). Também foi avaliada a infiltração gordurosa por meio da classificação de Goutallier, teve como resultado 11 pacientes sem evidências de infi-

Tabela 1 Agrupamento do Escore de Carter-Rowe

\begin{tabular}{|l|l|l|}
\hline & N & $\%$ \\
\hline$\leq 50$ (ruim) & 1 & 4 \\
\hline $51-74$ (regular) & 2 & 8 \\
\hline $75-89$ (bom) & 2 & 8 \\
\hline $90-100$ (excelente) & 20 & 80 \\
\hline Total & 25 & 100 \\
\hline
\end{tabular}

Tabela 2 Agrupamento do Escore de Walch-Duplay

\begin{tabular}{|l|l|l|}
\hline & N & $\%$ \\
\hline$\leq 50$ (ruim) & 0 & 0 \\
\hline $51-74$ (regular) & 2 & 8 \\
\hline $75-89$ (bom) & 10 & 40 \\
\hline $90-100$ (excelente) & 13 & 52 \\
\hline Total & 25 & 100 \\
\hline
\end{tabular}


Tabela 3 Comparação da força de rotação lateral em relação ao ombro contralateral

\begin{tabular}{|l|l|l|l|l|l|l|}
\hline & $\mathbf{N}$ & Média (em kg) & Desvio-padrão & Diferença média & IC 95\% & Valor $\boldsymbol{p}$ \\
\hline Lado operado & 25 & 8,96 & 2,67 & $-0,92$ & $(-1,44 ;-0,40)$ & $<0,001$ \\
\hline Lado não operado & 25 & 9,88 & 2,88 & & & \\
\hline
\end{tabular}

Tabela 4 Comparação da rotação lateral em 0。(RL1) e 90॰ (RL2) de abdução com ombro contralateral

\begin{tabular}{|l|l|l|l|l|l|l|}
\hline & $\mathbf{n}$ & Média (em graus) & Desvio-padrão & Diferença média & IC 95\% & Valor $\boldsymbol{p}$ \\
\hline RL1 lado operado & 25 & 77,04 & 14,6 & $-10,04$ & $(-14,74 ;-5,34)$ & $<0,001$ \\
\hline RL1 contralateral & 25 & 87,08 & 7,07 & & & \\
\hline RL2 lado operado & 25 & 94,52 & 12,37 & -10 & $(-14,26 ;-5,74)$ & $<0,001$ \\
\hline RL2 contralateral & 25 & 104,52 & 14,61 & & & \\
\hline
\end{tabular}
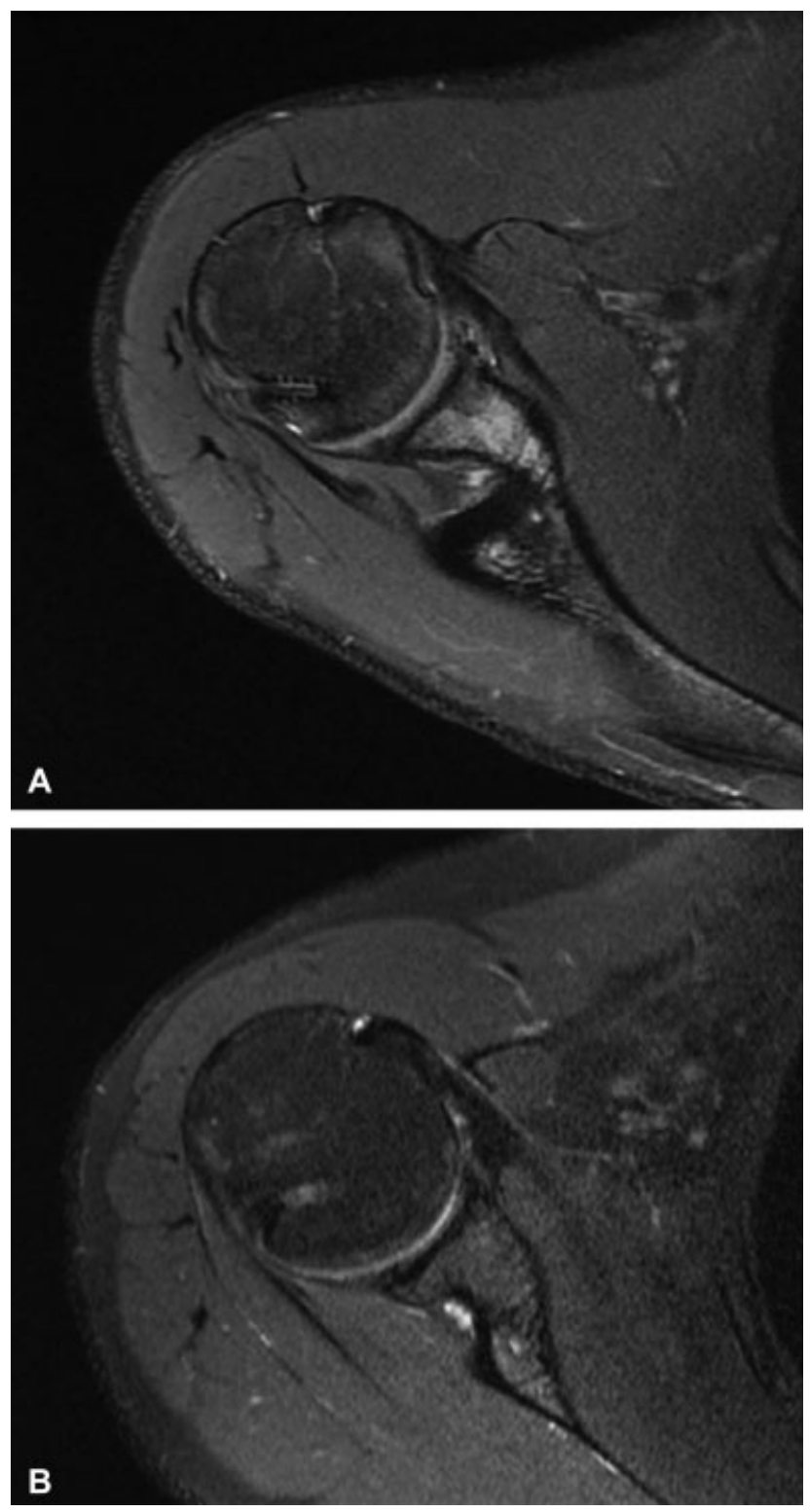

Fig. 4 (A) preenchimento parcial com defeito residual pequeno da lesão de Hill-Sachs visualizado pela ressonância magnética; (B) preenchimento completo. ltração gordurosa (estágio 0) e quatro com indícios de estrias de gordura (estágio 1).

\section{Discussão}

A técnica de remplissage tem sido usada na abordagem de lesões de Hill-Sachs off track na ausência de grandes lesões ósseas da glenoide, sempre associada ao reparo artroscópico da lesão de Bankart. Seu uso já apresenta amplo respaldo tanto do ponto de vista biomecânico ${ }^{13}$ como clínico, com resultados funcionais satisfatórios, baixos índices de recidiva e diminuição discreta da rotação lateral, sem repercussão funcional. ${ }^{14-21}$ Esses benefícios, somados aos seus índices de complicações menores do que $1 \%,{ }^{14}$ excluindo-se as recidivas, são consideravelmente inferiores aos dos procedimentos abertos, fazem com que o procedimento de remplissage seja uma boa opção no arsenal terapêutico da instabilidade anterior.

Em nosso grupo, usamos como protocolo para indicação da técnica de remplissage o índice de Hardy ${ }^{22}$ maior ou igual a 20\%, a lesão de Hill-Sachs é considerada off track, associado a perda óssea glenoidal menor do que $25 \%$.

Estudos prévios ${ }^{6,15-21}$ citam taxas de recidiva que variam de 0 a $12 \%$ com revisão sistemática que aponta média de $5,4 \%{ }^{14}$ Vale citar a dificuldade na comparação, haja vista diferenças na definição de recidivas. Alguns consideram falha do tratamento somente quando há luxação e outros o consideram quando a apreensão é positiva no pós-operatório. Em nossa casuística, quatro pacientes (16\%) apresentaram teste de apreensão positivo, somente um desses (4\%)

Tabela 5 Fisor

\begin{tabular}{|l|l|l|}
\hline Preenchimento & $\mathbf{n}$ & $\%$ \\
\hline Fisor 0 (ausência de preenchimento) & 0 & 0 \\
\hline Fisor 1 (preenchimento de baixo grau) & 0 & 0 \\
\hline Fisor 2 (preenchimento de baixo grau) & 0 & 0 \\
\hline Fisor 3 (preenchimento de alto grau) & 4 & 25 \\
\hline Fisor 4 (preenchimento de alto grau) & 12 & 75 \\
\hline
\end{tabular}


passou por episódio de subluxação no pós-operatório. Não houve recidiva de luxação nos indivíduos avaliados. Todos relataram estar satisfeitos com o resultado do tratamento cirúrgico. Dois pacientes apresentaram capsulite adesiva durante a reabilitação pós-operatória, com boa evolução.

Quanto à avaliação funcional objetiva, representada por escores funcionais, é predominante na literatura a presença de bons a excelentes resultados no pós-operatório, com médias do escore de Carter-Rowe de 83 a 91 pontos. ${ }^{15-19}$ Boileau et al. ${ }^{15}$ tiveram $87 \%$ de resultados bons a excelentes no escore de Walch-Duplay e Merolla et al. apresentaram 96\% de bons a excelentes resultados tanto para esse como para aquele escore. Obtivemos índices semelhantes com $88 \%$ de bons a excelentes resultados para o Carter-Rowe e 92\% para o Walch-Duplay.

Outro ponto controverso na literatura é o impacto da remplissage na rotação lateral do ombro. Estudos prévios mostram perda de 1,9 a 10 。 com relevância estatística ${ }^{15-17} \mathrm{e}$ outros mostram discretas perdas sem relevância estatística. ${ }^{14,19,21}$ Novamente, se faz difícil a comparação entre os diferentes trabalhos, pelas diferenças quanto a técnicas de medição, posição do ombro e comparação com lado contralateral. Conforme citado previamente, obtivemos resultados com perda estatisticamente significativa de rotação lateral de cerca de 10 ॰ tanto em RL1 como em RL2. Vale ressaltar que esse fato não vem acompanhado de prejuízo nas avaliações funcionais objetivas (escores) ou subjetivas (relato de satisfação com o tratamento).

Visto a abordagem do tendão infraespinal ser parte da técnica cirúrgica, optamos por avaliar seu possível déficit que influencia na força global de rotação lateral do ombro, medida em RL1. Também a infiltração gordurosa de seu ventre foi avaliada com ressonância magnética.

$\mathrm{Na}$ avaliação da força de rotação lateral, houve diferença significativa de aproximadamente $1 \mathrm{~kg}$ na medida quando comparada com o lado contralateral, diferentemente dos resultados de Merolla et al. ${ }^{17}$ e Zhu et al., ${ }^{18}$ que não encontraram diferença significativa na referida medida. Visto os escores funcionais não incluírem medida de força, seria pouco conclusiva a comparação da força com seus resultados.

A avaliação de imagem por meio de RM mostrou que todos os 16 pacientes que concordaram com o exame apresentaram, no máximo, estrias de gordura em seu ventre muscular (Goutallier 1), está em concordância com o resultado de Park et al. ${ }^{23}$ Quanto ao preenchimento da lesão de Hill-Sachs, o fato de todos os pacientes apresentarem preenchimento bom a excelente (alto grau de preenchimento) de acordo com adaptação feita pelo nosso grupo inviabilizou a análise estatística e sua comparação com função, amplitude de movimento ou força.

Rhee et al. ${ }^{12}$ concluíram que o Fisor é um critério de medição útil para avaliação dos resultados estruturais após o procedimento de remplissage. Foram encontrados $82,6 \%$ de bons/excelentes resultados no trabalho original, para 23 pacientes. Resultados regulares, pobres ou ausência de preenchimento foram vistos em $17,4 \%$ dos pacientes avaliados, sem correlação clínica. A classificação Fisor não foi usada na sua forma original no presente trabalho devido às variações para a $\mathrm{RM}$, ou seja, os exames foram feitos sem contraste e com todos os pacientes com o braço operado posicionado em rotação lateral com objetivo de retirar a tensão do tendão infraespinal e avaliar melhor o preenchimento do defeito de Hill-Sachs. Com a adaptação feita, obtivemos $100 \%$ de bons e excelentes resultados (preenchimento de alto grau), com discordância do trabalho original, conforme mencionado acima.

O presente estudo apresenta como limitações: 1) Quanto aos resultados de imagem, a avaliação comparativa da cicatrização e do preenchimento do defeito de Hill-Sachs ficou limitada devido à adaptação feita pelo nosso grupo em relação ao escore Fisor original. Essa adaptação se fez necessária devido aos protocolos usados para obtenção das imagens no pós-operatório serem diferentes; 2) Seguimento mínimo curto (12 meses), ausência de grupo controle e alto índice de perda de seguimento.

\section{Conclusão}

Nossa vivência com a técnica de remplissage apresentou bons a excelentes resultados funcionais (presentes em torno de $90 \%$ dos pacientes) com bons resultados quanto à cicatrização da capsulotenodese. Os resultados sugerem que não ter havido prejuízo morfológico ou funcional do músculo infraespinal, bem como que a discreta perda de rotação lateral não teve maiores repercussões funcionais. São necessários novos estudos com maior número de pacientes e investigação mais pormenorizada de sua prática esportiva para conclusões mais sólidas relacionadas a esses temas

Conflitos de Interesse

Os autores declaram não haver conflitos de interesse.

\section{Referências}

1 Hill HA, Sachs MD. The grooved defect of the humeral head: a frequently unrecognized complication of dislocations of the shoulder joint. Radiology 1940:690-700

2 Calandra JJ, Baker CL, Uribe J. The incidence of Hill-Sachs lesions in initial anterior shoulder dislocations. Arthroscopy 1989;5(04): 254-7

3 Spatschil A, Landsiedl F, Anderl W, Imhoff A, Seiler H, Vassilev I, et al. Posttraumatic anterior-inferior instability of the shoulder: arthroscopic findings and clinical correlations. Arch Orthop Trauma Surg 2006;126(04):217-22

4 Burkhart SS, De Beer JF. Traumatic glenohumeral bone defects and their relationship to failure of arthroscopic Bankart repairs: significance of the inverted-pear glenoid and the humeral engaging Hill-Sachs lesion. Arthroscopy 2000;16(07):677-94

5 Yamamoto N, Itoi E, Abe H, Minagawa H, Seki N, Shimada Y, et al. Contact between the glenoid and the humeral head in abduction, external rotation, and horizontal extension: a new concept of glenoid track. J Shoulder Elbow Surg 2007;16(05):649-56

6 Wolf EM, Pollack ME. Hill-Sachs "remplissage": an arthroscopic solution for the engaging Hill-Sachs lesion. Arthroscopy 2004;20 (Suppl 1):e14-5

7 Godinho GG, Souza JMG, Freitas JMA, Santos FML, Vieira AW, João FM. Tratamento da instabilidade anterior do ombro: experiência com a técnica de Morgan. Rev Bras Ortop 1997;32(04):265-71

8 Garcia GH, Degen RM, Liu JN, Kahlenberg CA, Dines JS. Accuracy of suture passage during arthroscopic remplissage-what anatomic landmarks can improve it?: a cadaveric study Orthop J Sports Med 2016;4(08):2325967116663497 
9 Rowe CR, Patel D, Southmayd WW. The Bankart procedure: a longterm end-result study. J Bone Joint Surg Am 1978;60(01):1-16

10 Walch G. Directions for the use of quotation of anterior instabilities of the shoulder. In: First Opne Congress of the Europe Society of Surgery of Jain NB, Wilcox RB 3 rd, Katz JN, Higgins LD(eds.). Clinical examination of the rotator cuff. PM R. 2013;5(1):45-56

11 Jain NB, Wilcox RB III, Katz JN, Higgins LD. Clinical examination of the rotator cuff. PM R 2013;5(01):45-56

12 Rhee YG, Cho NS, Yoo JH, Lee WG. Filling Index Score of Remplissage (FISOR): a useful measurement tool to evaluate structural outcome after remplissage. J Shoulder Elbow Surg 2015;24(04): 613-20

13 Hartzler RU, Bui CN, Jeong WK, Akeda M, Peterson A, McGarry M, et al. Remplissage of an off-track Hill-Sachs lesion is necessary to restore biomechanical glenohumeral joint stability in a bipolar bone loss model. Arthroscopy 2016;32(12):2466-76

14 Buza JA III, Iyengar JJ, Anakwenze OA, Ahmad CS, Levine WN. Arthroscopic Hill-Sachs remplissage: a systematic review. J Bone Joint Surg Am 2014;96(07):549-55

15 Boileau P, O'Shea K, Vargas P, Pinedo M, Old J, Zumstein M. Anatomical and functional results after arthroscopic Hill-Sachs remplissage. J Bone Joint Surg Am 2012;94(07):618-26

16 Haviv B, Mayo L, Biggs D. Outcomes of arthroscopic "remplissage": capsulotenodesis of the engaging large Hill-Sachs lesion. J Orthop Surg Res 2011;6:29

17 Merolla G, Paladini P, Di Napoli G, Campi F, Porcellini G. Outcomes of arthroscopic Hill-Sachs remplissage and anterior Bankart repair: a retrospective controlled study including ultrasound evaluation of posterior capsulotenodesis and infraspinatus strength assessment. Am J Sports Med 2015;43(02):407-14

18 Zhu YM, Lu Y, Zhang J, Shen JW, Jiang CY. Arthroscopic Bankart repair combined with remplissage technique for the treatment of anterior shoulder instability with engaging Hill-Sachs lesion: a report of 49 cases with a minimum 2-year follow-up. Am J Sports Med 2011;39(08):1640-7

19 Franceschi F, Papalia R, Rizzello G, Franceschetti E, Del Buono A, Panascì $M$, et al. Remplissage repair-new frontiers in the prevention of recurrent shoulder instability: a 2-year follow-up comparative study. Am J Sports Med 2012;40(11):2462-9

20 Gracitelli MEC, Helito CP, Malavolta EA, Ferreira Neto AA, Benegas E, Prada FS, et al. Results from filling "remplissage" arthroscopic technique for recurrent anterior shoulder dislocation. Rev Bras Ortop 20115;46(06):684-90

21 Garcia GH, Wu HH, Liu JN, Huffman GR, Kelly JD IV. Outcomes of the remplissage procedure and its effects on return to sports: average 5-year follow-up. Am J Sports Med 2016;44(05):1124-30

22 Hardy P, Lopes R, Bauer T, Conso C, Gaudin P, Sanghavi S. New quantitative measurement of the hill-sachs lesion: aprognostic factor for clinical results of arthroscopic glenohumeral stabilization. Eur J Orthop Surg Traumatol 2012;22:541-7

23 Park MJ, Garcia G, Malhotra A, Major N, Tjoumakaris FP, Kelly JD IV. The evaluation of arthroscopic remplissage by high-resolution magnetic resonance imaging. Am J Sports Med 2012;40(10): 2331-6 